\section{Anesthetic management of broncho- pleurocutaneous fistula - an alternate approach}

To the Editor:

A 48-yr-old man with a persistent left bronchopleurocutaneous fistula (BPCF) and empyema thoracis following pneumonectomy presented for bronchial stump closure and left thoracoplasty. The patient refused any attempts at awake intubation.

He was placed in semi-recumbent position with the left side down after topicalization and mask induction. Right-sided double-lumen tube (DLT) placement was attempted while the patient was breathing spontaneously, but was unsuccessful in the absence of adequate muscle relaxation. Attempts to position a left bronchial blocker under fibreoptic guidance resulted in the balloon either slipping into the fistulous opening or backing up into the trachea. Advancing a single-lumen tube into the right main stem bronchus resulted in right upper lobe obstruction and attempted DLT insertion over a tube exchanger was also unsuccessful.

When rescheduled, he was positioned and induced in the same manner as before and intubated with an 8.5 size endotracheal tube. Anesthesia was maintained with air, oxygen and sevoflurane using a Bain circuit, the patient breathing spontaneously. A triple-lumen central line and a transesopheal echocardiograph (TEE) were added to standard monitoring. The patient was positioned in the sitting position and stabilized in the appropriate frame, with his hands abducted and flexed forwards. The surgeon approached the BPCF through a left thoracotomy by transaxillary approach.

Transtracheal jet-ventilation was delivered through the tip of a catheter placed above the level of fistula, to lessen the possibility of barotrauma. Anesthesia was maintained with an infusion of ketamine. During jet-ventilation $\mathrm{PaO}_{2}$ values were 80 to $90 \mathrm{mmHg}$ while $\mathrm{PaCO}_{2}$ increased to $60 \mathrm{mmHg}$. The fistula was repaired and leak tested while purulent material from the left chest cavity was suctioned clean. Controlled ventilation restored blood gas levels towards normal. Left thoracoplasty was completed and the patient was extubated in the operating room. Postoperative pain control was achieved by a thoracic epidural catheter with continuous infusion of bupivacaine and fentanyl.

While anesthetizing the patient with BPCF in the sitting position, oxygenation can be assured with jetventilation, and gravity aids in draining away purulent secretions from the trachea. ${ }^{1} \mathrm{CO}_{2}$ accumulation can be managed by limiting the surgical time and estab- lishing controlled ventilation at the earliest opportunity. Hypotension can be corrected with crystalloid infusion. The hazard of air embolism can be monitored with TEE and treated with a triple lumen central venous catheter. ${ }^{2}$

When conventional methods of pulmonary isolation fail, anesthesia and surgery pose unusual challenges during the operative management of BPCF. ${ }^{3,4}$ Careful planning and meticulous anesthetic management can transform a difficult case into a manageable one.

Ramasamy Govindarajan MD

Ambarish Mathur MD

Judith Aransohn MD

Wagih Saweris MD

Biswajit Ghosh MD

Madhan Kumar Sathyamoorthy MD

The Brookdale University Hospital and Medical

Center, Brooklyn, USA

E-mail: gbiswajit@yahoo.com

\section{References}

1 Bishop MJ, Benson MS, Sato P, Pierson DJ. Comparison of high-frequency jet ventilation with conventional mechanical ventilation for bronchopleural fistula. Anesth Analg 1987; 66: 833-8.

2 Porter JM, Pidgeon C, Cunningham AJ. The sitting position in neurosurgery: a critical appraisal. $\mathrm{Br} \mathrm{J}$ Anaesth 1999; 82: 117-28.

3 Hollaus PH, Lax F, el-Nashef BB, Hauck HH, Lucciarini P, Pridun NS. Natural history of bronchopleural fistula after pneumonectomy: a review of 96 cases. Ann Thorac Surg 1997; 63: 1391-7.

4 James BE, Edmond C, Steven MN. Anesthesia for thoracic surgery. In: Barash $\mathrm{PG}$, Cullen BF, Stoelting RK (Eds). Clinical Anesthesia. Philadelphia LippincottRaven; 1997: 669-97.

\section{"The critical airway"}

To the Editor:

In their editorial of March 2005, Murphy et al. propose a new paradigm for the difficult airway (DA) approach in the operating room (OR) centred on ventilation and oxygenation rather than intubation. ${ }^{1}$ We extend this concept to the emergent airway management outside the OR and describe the "critical airway" (CA). The CA is defined as the airway management outside the OR in a patient requiring emergent oxygenation and ventilation secondary to pathology. It is the antithesis of the OR airway management as the 
situation is uncontrolled, tools are suboptimal and the patient, by definition, is critically ill.

Establishing an emergent airway in a remote location is "inherently difficult" as the routine airway assessment and management are changed producing failure rates much higher than would be acceptable in the "elective" setting. The OR paradigms of airway management need to be reassessed.

1) The anesthesiologist is not formally trained to manage the airway outside the OR. Predicting difficult laryngoscopic intubation in a critically ill unresponsive patient requiring emergency airway management is not practical as more than half of the clinical airway examination components suggested by the "Practice guidelines for management of the difficult airway" need a cooperative patient. ${ }^{2}$

2) The patient presents with acute comorbidities, a "full stomach", in immediate need for oxygenation and ventilation. A rapid sequence induction and intubation is not always indicated or applicable. ${ }^{3}$

3 ) The airway devices available are limited and the work environment suboptimal.

4) The time available to prepare and to successfully achieve ventilation and oxygenation is measured in seconds.

The goal of the CA management is efficient ventilation and oxygenation with no stomach inflation, regurgitation, and aspiration and without inducing comorbidities in an already critically ill patient (hypotension, hypertension, bradycardia, tachycardia, hypoxemia, hypercarbia, cardiac arrest, cervical injury). ${ }^{4,5}$

The inability to efficiently bag mask ventilate and the persistence to intubate a "full" stomach patient are at the core of most complications in the remote setting. ${ }^{3}$ The CA is a dynamic concept that demands the anesthesiologist to be skilled in "all four dimensions" of the airway management techniques: bag mask ventilation (BMV), supraglottic airway (SGA; laryngeal mask airway, Combitube, laryngeal tube...), glottic airway (GA; laryngoscopy, endotracheal intubation, Eschmann), or infraglottic airway (IGA; cricothyroid membrane or surgical techniques); any of the techniques can be used as the first option (Figure).

The CA receives minimal attention in the anesthesiology literature, during residency training or workshops. We use the CA concept to train our residents by emphasizing the differences from the OR routine. The ASA DA algorithm is a tool primarily used to avoid crisis in a controlled environment (OR); the CA concept applies to crisis in progress in a remote location. The classic "cannot intubate-cannot ventilate" becomes "cannot ventilate with BVM and a supra-
Rapid Clinical Assessment

Determination of Initial Airway Management Approach

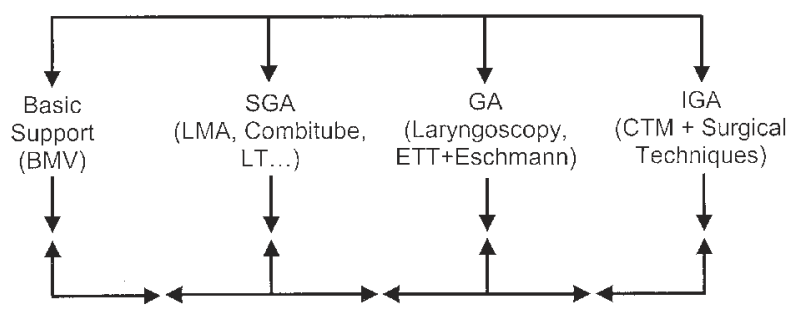

Failed Initial Attempt:

1. Can initial attempt be improved?

2. Rapid progression to Alternative Approach

FIGURE The management of the critical airway. $\mathrm{BMV}=$ bag mask valve; $S G A=$ supraglottic airway; $\mathrm{GA}=$ glottic airway; IGA = infraglottic airway

glottic device and cannot intubate after an optimal attempt".

It is ironic that when we should function at our best as "airway consultants," we relay on suboptimal or no airway assessment. Can we define predictors for airway management "difficulty" in "all four dimensions" outside the OR? Can we define optimal use of specific devices: BVM, SGA, GA, and IGA? How does the clinical status of the patient impact on our choice of airway devices?

As airway specialists, anesthesiologists should address through research and training the specific issues of the CA thus improving the outcome of the critically ill. We agree with Murphy et al. that we have "to change how we think about airway management" and to shift from intubation to oxygenation and ventilation. This principle applies both inside and outside the OR.

\author{
Adrian A. Matioc MD \\ George Arndt MD \\ University of Wisconsin Medical School, Madison, \\ USA \\ E-mail: aamatioc@facstaff.wisc.edu
}

\section{References}

1 Murphy M, Hung O, Launcelott G, Law A, Morris I. Predicting the difficult laryngoscopic intubation: are we on the right track? (Editorial). Can J Anesth 2005; 53: 231-5.

2 American Society of Anesthesiologists Task Force on Management of the Difficult Airway. Practice guide- 
lines for management of the difficult airway: an updated report by the American Society of Anesthesiologists Task Force on Management of the Difficult Airway. Anesthesiology 2003; 98: 1269-77.

3 Nolan JD. Prehospital and resuscitative airway care: should the gold standard be reassessed? Curr Opin Crit Care. 2001; 7: 413-21.

4 Mort TC. The incidence and risk factors for cardiac arrest during emergency tracheal intubation: a justification for incorporating the ASA guidelines in the remote location. J Clin Anesth 2004; 16: 508-16.

5 Schwartz DE, Matthay MA, Cohen NH. Death and other complications of emergency airway management in critically ill adults. A prospective investigation of 297 tracheal intubations. Anesthesiology 1995; 82: 367-76.

\section{"Keep out of trouble" airway algorithm}

To the Editor:

In his editorial, "Predicting the difficult laryngoscopic intubation: are we on the right track" ${ }^{1}$ Dr. Murphy asks a relevant question and I agree with his answer. In truth, every intubation is potentially difficult, suggesting that the current mystique surrounding the difficult airway is both exaggerated and beside the point. Early in my career, I noticed that critical incident reports about difficult airways usually involve "losing the airway" (this is not surprising: a "lost" airway is rapidly lethal so those patients may not reach our care). Therefore, not losing the existing airway should be the first goal of management. All of the techniques of intubation are much easier with stable gas exchange and a bit of time.

With this in mind, many years ago I developed an algorithm for teaching medical students how to quickly assess the airway for potential complications in order to avoid making a bad situation worse (Figure). This includes the "dimensions" suggested by Dr. Murphy plus a few more. Its strengths are: relative simplicity; a preventative point of view; and quick identification of situations where speed matters. In the last circumstance, admittedly uncommon, everyone has to develop his or her own best intubation technique (I favour one involving the lighted sytlet), and usually the option of surgical airway exists. If a surgical airway is not possible, then early identification is even more crucial.

Although this approach has not been compared in a randomized double-blind study with consensus approaches (they also have not been validated) due to the low incidence of events as noted by Dr. Murphy, I can only say that it has been uniformly successful in my practice.

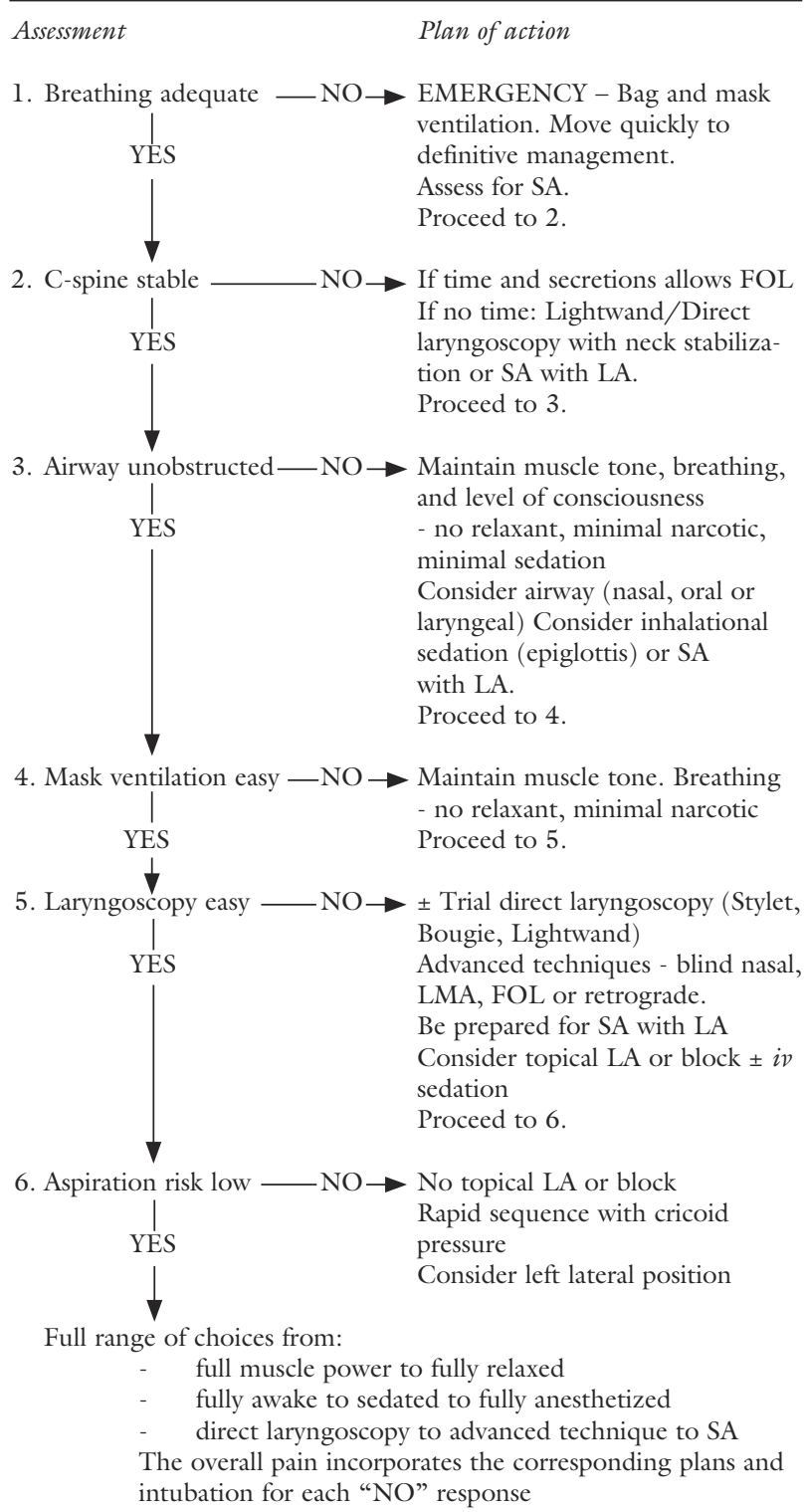

FIGURE K.O.O.T. (keep out of trouble) airway algorithm. An aide in choosing a safe technique of airway management. $\mathrm{SA}=$ surgical airway; $\mathrm{FOL}=$ fibreoptic laryngoscopy; $\mathrm{LA}=$ local anesthetic; LMA = laryngeal mask airway .

Laurence W. Lee MD FRCPC

Memorial University of Newfoundland, St. John's,

Canada

E-mail: lwlee@shaw.ca

\section{Reference}

1 Murphy M, Hung O, Launcelott G, Law A, Morris I. Predicting the difficult laryngoscopic intubation: are we on the right track? (Editorial). Can J Anesth 2005; 52: $231-5$. 